\title{
Effects of Pre-Workout Supplements on Strength, Endurance, and Mood
}

Anya C. Ellerbroek

Nova Southeastern University, ae359@mynsu.nova.edu

Jose Antonio

Nova Southeastern University, ja838@mynsu.nova.edu

Follow this and additional works at: https://nsuworks.nova.edu/ijahsp

Part of the Sports Sciences Commons

\section{Recommended Citation}

Ellerbroek AC, Antonio J. Effects of Pre-Workout Supplements on Strength, Endurance, and Mood. The Internet Journal of Allied Health Sciences and Practice. 2019 Jan 01;17(1), Article 7.

This Manuscript is brought to you for free and open access by the College of Health Care Sciences at NSUWorks. It has been accepted for inclusion in Internet Journal of Allied Health Sciences and Practice by an authorized editor of NSUWorks. For more information, please contact nsuworks@nova.edu. 


\title{
Effects of Pre-Workout Supplements on Strength, Endurance, and Mood
}

\begin{abstract}
Purpose: The purpose of this pilot study was to assess the acute effects of consuming pre-workout supplements on indices of muscular strength, endurance and mood states.

Methods: In a double- blind, placebo-controlled, randomized crossover design, fourteen moderate to highlytrained recreational athletes ( 7 female, 7 male) participated in this investigation. Subjects came to the lab twice between testing sessions. They consumed either pre supplement (mixed with 8 to 12 ounces of water) or placebo 30-minutes prior to testing. The pre-workout supplement combination (Athelite Nutrition Inc.) contained 15.62 grams per serving, $25 \mathrm{kcals}$, that consisted of a proprietary blend including caffeine (as green coffee bean extract), L-theanine, black pepper extract, micronized creatine monohydrate, CarnoSyn ${ }^{\circledR}$ beta-alanine, Huperzine A, N-Acetyl L-carnitine, Nitrosigine $\left.{ }^{\circledR}\right)$, or placebo. The placebo was a similar tasting drink with an equal amount of caffeine. Their body composition was assessed via the DEXA (Hologic Model Horizon W). Participants' mood was also assessed via a profile mood states questionnaire (POMS) 30 minutes after product or placebo was consumed. After taking the profile mood states questionnaire, subjects had their exercise performance assessed via the 1-repition maximum bench press followed by bench press repetitions to failure at $60 \%$ of 1-repetition maximum with 30 seconds rest between sets (3 total sets).

Results: There were significant differences $(p$

Conclusion: The results demonstrated that the acute consumption of pre-workout supplements can enhance muscular endurance. Caffeine alone cannot explain effect on muscular endurance since the placebo also contained caffeine. However, the supplements had no effect on strength or mood states.
\end{abstract}

\section{Author Bio(s)}

Anya Ellerbroek, B.A. Sports Exercise Science, CISSN, is the research assistant at the International Society of Sports Nutrition (ISSN) with Dr. Jose Antonio.

Dr. Jose Antonio PhD, FISSN, CSCS, is the president of the International Society of Sports Nutrition (ISSN), and faculty member of the Sports Exercise Science Bachelors- Human Health Science Department, at Nova Southeastern University in Ft. Lauderdale Fl.

\section{Acknowledgements}

We would like to thank Athelite Nutrition Inc. for providing the pre workout supplement. We would like to thank Jose Antonio for editing, proof reading, and guidance throughout the study. We would also like to thank all research assistants for their work during the study. The study was funded by Athletite Nutrition Inc. The authors do not have any competing interests. 


\title{
IIIAHSP \\ The Internet Journal of Allied Health Sciences and Practice \\ Dedicated to allied health professional practice and education
}

Vol. 17 No. 1 ISSN 1540-580X

\section{Effects of Pre-Workout Supplements on Strength, Endurance, and Mood: a Pilot Study}

\author{
Anya C. Ellerbroek \\ Jose Antonio
}

Nova Southeastern University

United States

\begin{abstract}
Purpose: The purpose of this pilot study was to assess the acute effects of consuming pre-workout supplements on indices of muscular strength, endurance and mood states. Methods: In a double- blind, placebo-controlled, randomized crossover design, fourteen moderate to highly-trained recreational athletes (7 female, 7 male) participated in this investigation. Subjects came to the lab twice between testing sessions. They consumed either pre-supplement (mixed with 8 to 12 ounces of water) or placebo 30 minutes prior to testing. The pre-workout supplement combination (Athelite Nutrition Inc.) contained 15.62 grams per serving, $25 \mathrm{kcals}$, that consisted of a proprietary blend including caffeine (as green coffee bean extract), L-theanine, black pepper extract, micronized creatine monohydrate, CarnoSyn ${ }^{\circledR}$ beta-alanine, Huperzine A, N-Acetyl L-carnitine, Nitrosigine ${ }^{\circledR}$ ), or placebo. The placebo was a similar tasting drink with an equal amount of caffeine. Their body composition was assessed via the DEXA (Hologic Model Horizon W). Participants' mood was also assessed via a profile mood states questionnaire (POMS) 30 minutes after product or placebo was consumed. After taking the profile mood states questionnaire, subjects had their exercise performance assessed via the 1-repition maximum bench press followed by bench press repetitions to failure at $60 \%$ of 1 repetition maximum with 30 seconds rest between sets ( 3 total sets). Results: There were significant differences $(p<0.05)$ between the supplement and placebo for the number of repetitions to failure as well as total weight lifted. However, there were no differences for any of the other parameters measured. Conclusion: The results demonstrated that the acute consumption of pre-workout supplements can enhance muscular endurance. Caffeine alone cannot explain effect on muscular endurance because the placebo also contained caffeine. However, the supplements had no effect on strength or mood states.
\end{abstract}

Keywords: endurance; caffeine, pre-workout; supplement 


\section{INTRODUCTION}

Pre-workout supplements have gained in popularity with recreational and professional athletes for the purpose of improving exercise performance in the last few years. ${ }^{1-3}$ The popularity for these supplements has increased the demand for companies to develop new, effective, proprietary blends that do not rely on caffeine to be the main ergogenic aid. The consumption of multiple ingredient products has shown to increase workout performance. ${ }^{3,4}$

The most popular proprietary ingredients in pre-workout supplements contain caffeine as the main ingredient. ${ }^{5}$ Its effectiveness as an ergogenic aid in improving performance, power, endurance, focus, and concentration has been well established. ${ }^{4-8}$ Other popular proprietary ingredients in pre-workout supplements include creatine, beta alanine, carnosine, L-theanine, L-carnitine, and leucine. Creatine is a widely studied ergogenic aid shown to replenish ATP stores quickly aiding in recovery, speed, increasing strength, and helps improving body composition. ${ }^{9}$ Beta alanine, a precursor to carnosine, has shown to increase performance during high intensity training, increase performance overall, and increase lean body mass. ${ }^{10,11}$ In addition, beta alanine has shown to aide recovery time, allowing for repeated bouts of high performance output. ${ }^{12,13 C a r n o s i n e ~ f u n c t i o n s ~ a s ~ a n ~ a c i d ~ b u f f e r ~ i n ~ t h e ~}$ body, improving time to fatigue. ${ }^{11}$ L-theanine, an amino acid, primarily found in the tea plant, camellia senisis, is known to promote relaxation while increasing alertness and focus, and has recently been added to pre-workout supplements because the combination of it with caffeine has shown to increase performance, especially in tasks that require speed and accuracy. ${ }^{14,15}$ The amino acid Lcarnitine in the form of $\mathrm{N}$-acetyl L-carnitine has been demonstrated to increase alertness and aiding with recovery when combined with other ingredients. ${ }^{16}$ The essential amino acid leucine, has been demonstrated to stimulate protein synthesis in muscle post exercise, and may decrease muscle protein breakdown during and following exercise. 17,18

Additional but lesser known or used ingredients include black pepper, huperzine-A, and Nitrosigine®. Black pepper has been added due to some evidence showing that it enhances absorption of supplements. . $^{19,20}$ Huperzine-A is a lesser-known and not yet widely utilized ingredient. Huperzine-A, a compound extracted from plants, such as toothed clubmoss, is mostly researched acting as an acetylcholinesterase inhibitor, which increases the levels of the neurotransmitter acetylcholine. ${ }^{21}$ Acetylcholine helps to increase muscle contractions, and also raising cognitive function and alertness. ${ }^{22}$ Nitrosigine 8 is a patented mixture of the amino acid arginine, inositol, and potassium silicate..$^{23}$ The arginine-silicate mixture has been shown to increase blood flow, increase performance, delay fatigue, and aid in recovery. ${ }^{23,24}$ To measure athletes' mood states, as well as athletic performance, the Profile Mood States (POMS) questionnaire is widely used with the athletic population. ${ }^{25}$ The short version POMS 2 consists of 35 descriptive words to assess feelings, such as fatigue-inertia, tension-anxiety, and vigor-activity. Each item is scored from 0 "not at all" up to 4 "extremely".26

There is limited data comparing a multiple-ingredient pre-workout supplement with a placebo containing caffeine, and their effects on performance. It is hypothesize that acute ingestion of the multi-ingredient pre-workout supplement will significantly increase muscular strength and endurance, as well as mood states, compared to the caffeine-containing placebo. A randomized, doubleblind, placebo-controlled crossover design was utilized in this investigation.

\section{METHODS}

\section{Participants}

Fourteen healthy, moderate to highly-trained recreational athletes ( 7 males $/ 7$ females) (age mean $\pm S D=33.1 \pm 5.7$ years; height= $171.1 \pm 9.1 \mathrm{~cm}$; body mass $=78.8 \pm 21.9 \mathrm{~kg}$ ) participated in this investigation (Table 1). All participants regularly consumed caffeinecontaining products and exercised regularly. All procedures were approved by Nova Southeastern University's Institutional Review Board for the protection of human subjects. Subjects signed a written consent form prior to any supplementation or testing.

Table 1. Physical Characteristics

\begin{tabular}{|l|l|}
\hline Characteristic & Mean \pm SD \\
\hline Age yr & $33.1 \pm 5.7$ \\
\hline Height $\mathrm{cm}$ & $171.1 \pm 9.1$ \\
\hline Weight kg & $78.8 \pm 21.9$ \\
\hline BMC kg & $2.8 \pm 0.5$ \\
\hline Fat Mass kg & $18.7 \pm 8.3$ \\
\hline Lean Mass kg & $57.4 \pm 17.2$ \\
\hline \% Body Fat & $23.9 \pm 7.0$ \\
\hline
\end{tabular}

There were no significant differences between groups.

\section{Protocol}


Resistance-trained subjects were recruited from the local community (i.e., convenience sample of trained participants). In a doubleblind, placebo-controlled, crossover trial, participants were asked to come to the lab twice with at least seven days between testing sessions. Participants arrived at the lab 3 hours after fasting with no prior exercise that day. All subjects consumed the supplements or placebo (mixed with 8 to 12 ounces of water) 30 minutes prior to testing. Neither the participant nor the research assistant knew which product they were receiving. Body composition was assessed by DEXA (Hologic Model Horizon W). All testing was performed in accordance with the manufacturer's instructions. Subjects were asked to take off any jewelry, metal, and shoes prior to body composition testing. All testing was done with each subject at the same time of day (plus or minus 1 hour). Each scan lasted approximately 7 minutes. Thirty minutes after consuming the supplements or placebo, subjects were asked to fill out the profile of mood states questionnaire (POMS) on a computer located in the lab. The research assistant recorded the results. Performance testing included the 1-RM for the bench press and bench press repetitions to failure at $60 \%$ of 1-repition maximum (RM) with 30-second rests between sets (3 total sets). Subjects performed a movement-specific warm up on the bench press (i.e., $\sim 3$ sets of the bench press at 50 to $75 \%$ of one's estimated 1-RM). All performance testing was conducted by a strength and conditioning specialist (CSCS) who followed the NSCA's guide to tests and assessments. ${ }^{27}$ Participants were familiar with the performance test prior to study.

\section{Product}

The pre-workout supplement (Athelite Nutrition Inc.) contained 15.62 grams per serving, $25 \mathrm{kcals}$, that consisted of a proprietary blend including caffeine (as green coffee bean extract), L-theanine, black pepper extract, micronized creatine monohydrate, CarnoSyn $®$ beta-alanine, Huperzine A, N-Acetyl L-carnitine, and Nitrosigine®:

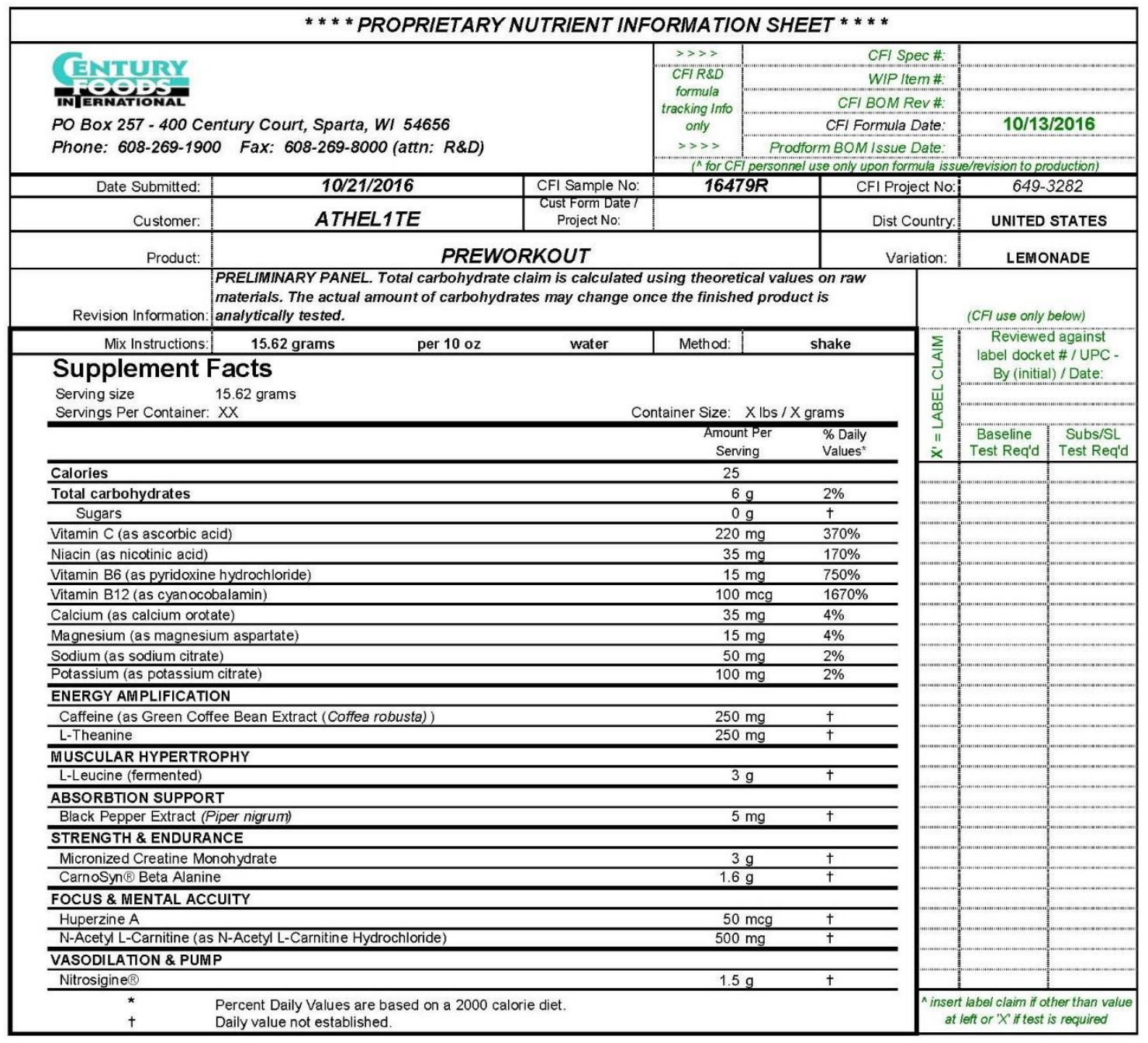

The placebo was similar tasting drink (Crystal Light) with the same amount of caffeine (250mg). 


\section{Statistics}

All data are expressed as the mean $\pm S D$. A paired t-test was used to assess the difference between the treatment and placebo conditions ( $p$ value less than 0.05 as significant).

\section{RESULTS}

There were significant differences $(p<0.05)$ between the supplement and placebo for the number of repetitions to failure as well as total weight lifted (Table 2). However, there were no differences for any of the other parameters measured (Table 3).

Table 2. Exercise Performance

\begin{tabular}{|l|l|l|}
\hline \multicolumn{1}{|c|}{ Activity } & \multicolumn{1}{c|}{ Placebo } & \multicolumn{1}{c|}{ Treatment } \\
\hline $1-\mathrm{RM} \mathrm{kg}$ & $94.0 \pm 52.2$ & $92.5 \pm 52.3$ \\
\hline Repetitions to Failure & $19.0 \pm 4.7$ & $22.7 \pm 4.0^{(\mathrm{a})}$ \\
\hline Total Weight Lifted $^{+}$ & $1124.2 \pm 739.3$ & $1290.4 \pm 818.3^{(\mathrm{b})}$ \\
\hline
\end{tabular}

Data are expressed as the mean \pm SD. Legend: $\mathrm{kg}-$ kilograms. Treatment significantly different than placebo, ${ }^{(b)} p=0.0190$. ${ }^{(a)} p=0.0131$. Total weight lifted was calculated by the number of repetitions times the weight lifted.

Table 3. Profile of Mood States

\begin{tabular}{|l|l|l|}
\hline \multicolumn{1}{|c|}{ Mood State } & \multicolumn{1}{c|}{ Placebo } & \multicolumn{1}{c|}{ Treatment } \\
\hline Anger & $3.3 \pm 5.1$ & $1.6 \pm 2.8$ \\
\hline Confusion & $3.4 \pm 2.3$ & $3.5 \pm 2.3$ \\
\hline Depression & $1.4 \pm 2.7$ & $1.3 \pm 2.9$ \\
\hline Fatigue & $3.4 \pm 4.7$ & $2.1 \pm 2.1$ \\
\hline Tension & $6.3 \pm 4.5$ & $7.4 \pm 6.0$ \\
\hline Vigor & $17.6 \pm 6.9$ & $16.9 \pm 7.4$ \\
\hline
\end{tabular}

Data are expressed as the mean $\pm S D$. There were no significant differences between groups.

\section{DISCUSSION}

Acute ingestion of the pre-workout supplement demonstrated enhanced muscular endurance; however, it had no effect on strength and mood states. This examination was the first to date comparing a pre-workout supplement to a placebo with both containing the same amount of caffeine. The $250 \mathrm{mg}$ of caffeine alone cannot explain the enhanced muscular endurance, because it was given to both groups. Previous research on multi-ingredient pre-workout supplements containing caffeine also showed a significant increase in muscular endurance. ${ }^{28}$ Spradley et al had twelve recreational trained males participating in a three week randomized, double-blind, placebo-controlled cross-over design study, to determine the effects of a pre-workout supplements on upper and lower body muscular endurance, aerobic, and anaerobic capacity, and choice reaction time. ${ }^{28}$ The pre-workout supplement ingredients included caffeine, beta alanine, branched chain amino acids, creatine, citruline, vitamin B-6, and B-12. The results showed significant improvements for the supplement group versus the placebo group in lower body muscular endurance, improved agility choice reaction performance, and an increase in perceived energy.

Jagim et al tested the acute ingestion of a multi-ingredient pre-workout supplement on strength performance, lower body power, and anaerobic capacity. ${ }^{29}$ The results of this double-blind, randomized, placebo controlled, crossover study showed a significant increase in upper body muscular endurance, fatigue, and alertness as well as increasing mean power output during anaerobic capacity sprint test. ${ }^{29}$ Caffeine is known to stimulate the central as well as the peripheral nervous system, improving time to exhaustion, alertness, and focus during endurance exercise. ${ }^{30,31}$ The effects of caffeine on endurance, even in small dosages may be related its glycogen sparing effect, while increasing free fatty acid utilization, as well as increasing the secretion of $\beta$ endorphins. ${ }^{32,33}$ The ISSN position stand on caffeine does, however, suggest that caffeine in anhydrous form versus a cup of caffeinated coffee exhibits greater benefits on endurance. ${ }^{31}$

Graham et al, investigating the effects of a placebo, decaffeinated coffee, caffeinated coffee (4.45mg caffeine $/ \mathrm{kg}$ ), decaffeinated coffee plus caffeine $(4.45 \mathrm{mg}$ caffeine $/ \mathrm{kg}$ ), and a caffeinated capsule $(4.45 \mathrm{mg}$ caffeine $/ \mathrm{kg})$ showed a significant increase in endurance only with the caffeinated capsule versus all other variables tested. ${ }^{34}$ The current investigation may have had to include a third testing date to include a placebo without caffeine to compare it with the product and the placebo containing caffeine. The significant increase in endurance with the product could be the results of the additional ingredients. 
The research results of caffeine and its effects on mood states have shown an increase in alertness, energy, clear mindedness, and anxiety. ${ }^{35}$ During the Peeling et al randomized, blind, crossover design investigation, subjects were assigned to either caffeine or placebo group, separated by one week of testing, and attend a 75-minute lecture on exercise rehabilitation sixty minutes after consumption of either caffeine or placebo. ${ }^{35}$ Following the lecture, subjects had to complete a mood perception questionnaire to assess perceived mood states, arousal, and focus during the lecture. The results showed a significant increase in energy, alertness, energetic, and anxiety when subjects had consumed caffeine. They were also better able to concentrate, and showed an increase in arousal with caffeine..$^{35}$

The Penetar et al study on the effects of caffeine on cognitive performance, mood, and alertness in sleep deprived humans using POMS and VAS (visual analog scales) also showed that caffeine was effective in increasing vigor, and decreasing feelings of fatigue and confusion. ${ }^{36}$ The possible explanation of not seeing any significant changes in mood states in the current investigation may be because both treatment and placebo supplements contained the same amount of caffeine. This examination would have to be conducted with an additional testing day adding a caffeine free placebo to assess significant mood state changes.

Creatine monohydrate, the most researched supplement to date, is known to improve body composition and significantly enhance exercise performance, especially anaerobic power, as a result of its ability to replenish ATP stores, allowing for a quick recovery after high intensity training. ${ }^{9}$ Long -term use (30 days and longer of $6 \mathrm{~g} /$ day) of creatine has also shown to reduce the extent of muscle damage and increase muscle force, whereas acute ingestion did not show any improvements. ${ }^{37}$ Creatine is used in many multi-ingredient pre-workout supplements to enhance performance, but creatine does require a loading period, which may explain why this investigation did not see significant changes in strength. ${ }^{28}$

Beta- alanine by itself is not regarded as an ergogenic aid. It is, however, used in many multi-ingredient supplements because it is known as the rate limiting amino acid for muscle carnosine, which has shown to improve high intensity performance. 12,39 Carnosine helps to buffer the acidity produced during high intensity training, thereby delaying muscular fatigue, and aid in recovery. ${ }^{39}$ Betaalanine supplementation does necessitate a period of chronic loading dosing (2g or less of a total $4-6 \mathrm{~g}$ daily for 2- 4 weeks) to increase muscle carnosine and experience maximal benefits. ${ }^{40} \mathrm{Hoffman}$ et al researched the effects of beta -alanine plus creatine, and creatine alone on strength, body composition, and endocrine changes during a 10-week resistance training program in collegiate football players, and found significant changes in strength, as well as body composition changes in the creatine plus beta alanine group . ${ }^{41}$ Because the current study investigated a one-time acute effect of the pre-workout supplement, it did not give subjects a supplement loading period required for beta-alanine to affect performance changes, especially in strength.

L-theanine is a non-dietary amino acid primarily found in the tea plant, camellia sinensis. It acts as a neurotransmitter in the brain, similar to glutamate and GABA, and it was found to cross the blood-brain barrier after 30 minutes of ingestion.42 It is known to promote a feeling of relaxation while feeling alert at the same time..$^{14}$ Owen et al, assessing $50 \mathrm{mg}$ caffeine combined with and without $100 \mathrm{~m} \mathrm{L-theanine} \mathrm{and} \mathrm{its} \mathrm{effect} \mathrm{on} \mathrm{mood} \mathrm{states,} \mathrm{has} \mathrm{shown} \mathrm{to} \mathrm{significantly} \mathrm{improve} \mathrm{performance} \mathrm{on} \mathrm{speed} \mathrm{and} \mathrm{accuracy} \mathrm{in}$ attention-switching tasks after 60 to 90 minutes post ingestion, versus caffeine and a placebo. ${ }^{15}$ The combination of $40 \mathrm{mg}$ caffeine and $97 \mathrm{mg} \mathrm{L-theanine} \mathrm{was} \mathrm{also} \mathrm{shown} \mathrm{to} \mathrm{increase} \mathrm{subjective} \mathrm{alertness} \mathrm{and} \mathrm{reduce} \mathrm{self-reported} \mathrm{tiredness} \mathrm{in} \mathrm{a} \mathrm{study} \mathrm{investigating}$ cognitive performance, alertness, blood pressure, and heart rate..$^{14}$

The present study did not find any significant changes in mood states after 30 minutes of ingesting the pre-workout supplement. It is possible that the time period from drinking the supplement to taking the POMS test was not enough to fully experience the effects of L-theanine and caffeine. Most studies on L-theanine and caffeine have subjects perform 40, 60, or 90 minutes after ingestion. ${ }^{43}$

The present study is also one of the first pre-workout supplement investigation on L-theaninie and caffeine combination. ${ }^{16}$ The ingredient L-carnitine is an amino acid synthesized in the liver from amino acids lysine and methionine, and its main function is to convert fat into energy. Most studies on acetyl L-carnitine have been performed on patients with onset of dementia and on Alzheimer patients to show its benefits on memory and brain function. ${ }^{44,45,46}$ It has been hypothesized that carnitine can improve performance through enhanced muscle fatty acid oxidation, delaying muscular fatigue, improve glucose homeostasis. ${ }^{46}$

Schields et al investigated a stimulant-free multi-ingredient pre-workout supplement containing acetyl-L-carnitine, tyrosine, alphaGPC, gingko biloba, L-theanine, blueberry extract, and their effects on alertness, focus, calmness, motivation, cognition, reaction, motor reaction, memory, and vertical jump power. ${ }^{16}$ The randomized, double-blind crossover design study compared the product to caffeine and placebo. The stimulant free product did increase alertness, motivation, cognition, memory, vertical jump and calmness, while the caffeine also saw an increase in all, except calmness. More research needs to be done on the effects of $\mathrm{N}$ acetyl L-carnitine on performance and cognition in healthy, active individuals. 
Huperzine-A is extracted from the Chinese herb in the Huperziceae family. It is known as inhibiting the breakdown of acetylcholine, thereby increasing the acetylcholine neurotransmitter involved in muscle contraction, and has been primarily studied in Alzheimer patients for its effect on memory and learning. ${ }^{22,47}$ There are no current investigations on Huperzine-A as an ingredient in preworkout supplement on its effects on performance and focus.

Nitrosigine ${ }^{\circledR}$ is a patented compound of inositol-stabilized arginine silicate that has shown to enhance performance by increasing focus and energy levels, as well as promoting blood flow to the muscles. Nitrosigine ${ }^{\circledR}$ has been demonstrated to increase arginine blood levels up to six hours post ingestion, as well as nitric oxide levels. ${ }^{23}$ Rood-Ojalvo et al studied the effects of Nitrosigine $\circledast$ in 16 healthy adult male subjects in a double-blind, placebo-controlled crossover study design using POMS scores to assess vigoractivity, fatigue-inertia, blood flow measurements, leg circumference measurements, and biomarkers of muscle recovery (creatinekinase and lactate dehydrogenase). ${ }^{23}$ The men had to take the supplement for 4 days (1500mg/day) and had to perform an intense leg exercise protocol on day one and day four, repeating the protocol after a week without any supplement or placebo. The results demonstrated a significant increase in energy measurements using the POMS vigor-activity after 72 hours and a significant decrease in fatigue-inertia from pre ingestion compared to the placebo. Blood flow measures significantly increased after day 3 , and creatine kinase levels decreased 72 hours post exercise compared to the placebo group.

In another randomized, prospective, double-blind study to evaluate cognitive effects of $1500 \mathrm{mg}$ of Nitrosigine $₫$, subjects consumed the product or placebo for 14 days and for a shorter period of 3 days. After 14 days and 3 days of dosing, subjects demonstrated enhanced processing speed in a Trail Making Test, as well as a reduction in completion times, with positive effects already seen after 10 minutes post ingestion. ${ }^{49}$

The current study did not find any significant changes in measures of vigor-activity and fatigue-inertia after 30 minutes of ingesting the product. A possible explanation may be that the dosage in the supplement is too low to enhance blood flow and energy levels. While ingestion in this multi-ingredient pre-workout supplement have shown results in previous studies on strength and mood states, our research could only find significant effects on muscular endurance after acute ingestion.

\section{LIMITATIONS}

Some limitations of this investigation can be attributed to the fact that proprietary blend supplements do not list the dose of each ingredient, which makes it impossible to identify which ingredient/s, other than caffeine, contributed to the improved endurance capacity. Beta alanine and creatine, both active ingredients in this product, require loading periods of several weeks (1 to 4 weeks) to enhance strength and power. Another limitation of this investigation relates to the subjects' characteristics. Males and females with moderate to high levels of training experience participated, which may have affected the statistical data, especially on strength.

\section{CONCLUSION}

The results of this study demonstrated that the acute consumption of a pre-workout supplement can enhance muscular endurance; however, it has no effect on strength or mood states. Further studies need to be done on the ingredients that affect mood states, concentration, and energy levels. The 30-minute post ingestion mood state testing may not have been enough time to fully affect significant changes.

\section{CONFLICT OF INTEREST}

Athelite Nutrition Inc. provided the pre-workout supplement. The study was funded by Athletite Nutrition Inc.

\section{REFERENCES}

1. Young CR, Stephens MB. Sports and nutritional supplement use in USMC recruits: a pilot study. Mil Med. 2009 Feb;174(2):158-61. [PMID: 19317196]

2. Erdman KA, Fung TS, Doyle-Baker PK, Verhoef MJ, Reimer RA. Dietary supplementation of high-performance Canadian athletes by age and gender. Clin J Sport Med. 2007 Nov;17(6):458-64. [PMID: 17993788]

3. Hoffman JR, Kang J, Ratamess NA, Hoffman MW, Tranchina CP, Faigenbaum AD. Examination of a pre-exercise, highenergy supplement on exercise performance. J Int Soc Sports Nutr. 2009;6:2. [PMID: 19126213].

4. Graham TE, Rush JW, van Soeren WH. Caffeine and exercise: metabolism, endurance and performance. Can J Appl Physiol. 1994;19(2):111-38. [PMID: 8081318]

5. Smith AE, Fukuda DH, Kendall KL, Stout JR. The effects of a pre-workout supplement containing caffeine, creatine, and amino acids during three weeks of high-intensity exercise on aerobic and anaerobic performance. J Int Soc Sports Nutr. 2010;7:10. [PMID: 20156347]

6. Doherty M, Smith PM. Effects of caffeine ingestion on exercise testing: a meta-analysis. Int J Sport Nutr Exerc Metab. 2004;14:626-46. [PMID: 15657469] 
7. Sokmen B, Armstrong LE, Kraemer WJ, Casa DJ, Dias JC, et al Caffeine use in sports: considerations for the athlete. $J$ Strength Cond Res. 2008;22(3):978-86. [PMID: 18438212]

8. Ganio MS, Klau JF, Casa DJ, Armstrong LE, Maresh CM. Effects of caffeine on sport-specific endurance performance: a systematic review. J Strength Cond Res. 2009;23(1):315-24. [PMID: 19077738]

9. Buford TW, Kreider RB, Stout JR, Greenwood M, Campbell B, et al International society of sports nutrition position stand: creatine supplementation and exercise. J Int Soc Sports Nutr. 2007;4:6. [PMID: 17908288]

10. Kreider RB, Wilborn CD, Taylor L, Campbell B, Almada AL, et al ISSN exercise \& sport nutrition review: research \& recommendations. J Int Soc Sports Nutr. 2010;7:7. [PMCID: PMC2853497]

11.Smith AE, Walter AA, Graef JL, Kendall KL, Moon JR, et al Effects of $\beta$-alanine supplementation and high-intensity interval training on endurance performance and body composition in men; a double-blind trial. J Int Soc Sports Nutr. 2009;6:5. [PMID: 19210788].

12. Trexler E, Smith-Ryan A, Stout J, Hoffman J, Wilborn C, et al International society of sports nutrition position stand: BetaAlanine. J Int Soc Sports Nutr. 2015;12:30. [PMID: 26175657]

13. Derave W, Ozdemier MS, Harris RC, Pottier A, Reyngoudt H, et al B-alanine supplementation augments muscle carnosine content and attenuates fatigue during repeated isokinetic contraction bouts in trained sprinters. J Appl Physiol. 2007;103(5):1736-43. [PMID: 17690198]

14. Giesbrecht T, Rycroft, JA, Rowson MJ, De Bruin EA. The combination of L-theanine and caffeine improves cognitive performance and increases subjective alertness. Nutr Neurosci. 2010;13(6):283-90. [PMID: 21040626]

15. Owen G, Parnell H, De Bruin EA, Rycroft JA. The combined effects of L-theanine and caffeine on cognitive performance and mood. Nutr Neurosci. 2008;11(4):193-8. [PMID: 18681988]

16. Shields K, Silva J, Rauch J, Lowery R, Ormes JA, et al The effects of a multi-ingredient cognitive formula on alertness, focus, motivation, calmness and psychomotor performance in comparison to caffeine and placebo. J Inter Soc Sports Nutr. 2014;11(Suppl 1):45. [PMCID: PMC4271648]

17. Mero A. Leucine supplementation and intensive training. Sports Med. 1999;27(6):347-58. [PMID: 10418071]

18. Pasiakos S, McClung H, McClung J, Margolis L, Andersen NE,et al Leucine-enriched essential amino acid supplementation during moderate steady state exercise enhances postexercise muscle protein synthesis. Am J Clin Nutr. 2011;94(3):809-18. [PMID: 21775557]

19. Badmaev V, Majeed M, Norkus EP. Piperine, an alkaloid derived from black pepper increases serum response of betacarotene during 14-days of oral beta-carotene supplementation. Nutr Res. 2000;19(3):381-8.

20. Badmaev V, Majeed M, Prakash L. Piperine derived from black pepper increases the plasma levels of coenzyme Q10 following oral supplementation. J Nutr Biochem. 2000;11(2):109-13. [PMID: 10715596]

21. Bolton TB, Lim SP. Action of acetylcholine on smooth muscle. Z Kardiol. 1991;80(Suppl 7):73-7. [PMID: 1665266]

22. Hai-yan Zhang. New Insights into hyperzine A for the treatment of Alzheimer's disease. Acta Pharmacoliga Sinica. 2012;33(9):1170-5. [PMID: 22941287]

23. Rood-Ojalvo S, Sandler D., Veledar E., Komorowski J. The benefits of inositol-stabilized arginine silicate as a workout ingredient. J Int Soc Sports Nutr. 2015;12(Suppl 1):14. [PMCID: PMC4595023]

24. Kalman D. Harvey PD, Ojalvo S, Komorowski J. Randomized prospective double-blind studies to evaluate the cognitive effects of inositol-stabilized arginine silicate in healthy physically active adults. Nutrients. 2016;8(11). pii e736. [PMID: 27869715]

25. Beedie C, Terry P, Lane AM. The profile of mood states and athletic performance: two meta-analyses. J App/ Sport Psych. 2000;12(1): 49-68.

26. McNair DM, Lorr M, Droppelman LF. Manual for the Profile of Mood States. San Diego, CA: Educational and Industrial Testing Service. 1971.

27. Triplett TN, Williams C, McHenry P, Doscher M. Strength and conditioning professional standards and guidelines. National Strength and Conditioning Association. Strength and Conditioning Journal. 2017;39(6):1-24. Available at: https://www.nsca.com/uploadedFiles/NSCA/Resources/PDF/Education/Tools_and_Resources/NSCA_strength_and_conditi oning_professional_standards_and_guidelines.pdf. Accessed August 11. 2018.

28. Spradley BD, Crowley K, Tai CY, Kendall K, Fukuda DH, et al Ingesting a pre-workout supplement containing caffeine, Bvitamins, amino acids, creatine, and beta-alanine before exercise delays fatigue while improving reaction time and muscular endurance. Nutr Metab. 2012;9:28. [PMID: 22463603]

29. Jagim AR, Jones MT, Wright GA, St Antoine C, Kovacs C, Oliver JM. The acute effects of multi-ingredient pre-workout ingestion on strength performance, lower body power and anaerobic capacity. J Int Soc Sports Nutr. 2016;13:11. [PMID: 26962303]

30. Roberts MD, Taylor LW, Wismann JA, Wilborn CD, Kreider RB, Willoughby DS. Effects of ingesting JavaFit Energy Extreme functional coffee on aerobic and anaerobic fitness markers in recreationally-active coffee consumers. J Int Soc Sports Nutr. 2007;4:25. [PMID: 18067677] 
31. Goldstein ER, Ziegenfuss T, Kalman D, Kreider R, Campbell B, et al International society of sports nutrition position stand: caffeine and performance. J Int Soc Sports Nutr. 2010;7(1):5. [PMID: 20205813]

32. Pasman WJ, van Baak MA, Jeukendrup AE, de Haan A. The effect of different dosages of caffeine on endurance and performance. Int J Sports Med. 1995;16(4):225-30. [PMID: 7657415]

33. Laurent D, Schneider KE, Prusaczyk WK, Franklin C, Vogel SM, et al Effects of caffeine on muscle glycogen utilization and the neuroendocrine axis during exercise. J Clin Endocrinol Metab. 2000;85(6):2170-5. [PMID: 1085248]

34. Graham TE, Hibbert E, Sathasivam P. Metabolic and exercise endurance effects of coffee and caffeine ingestion. J Appl Physio. 1998;85(3):883-9. [PMID: 9729561]

35. Peeling $P$, Dawson $B$. Influence of caffeine ingestion on perceived mood states, concentration, and arousal levels during a 75-min university lecture. Adv Physiol Educ. 2007;31(4):332-5. [PMID: 18057405]

36. Penetar DM, McCann U, Thorne D, Schelling A, Galinski C, et al Effects of caffeine on cognitive performance, mood, and alertness in sleep-deprived humans. Inst Med (US) Committee on Military Nutrition Research. Washington D.C. National Academies Press. 1994.

37. Rosene J, Matthews T, Ryan C, Belmore K, Bergsten A, et al Short and long-term effects of creatine supplementation on exercise induced muscle damage. J Sports Sci Med. 2009;8(1):89-96. [PMID: 24150561]

38. Hill CA, Harris RC, Kim HJ, Harris BD, et al Influence of beta-alanine supplementation on skeletal muscle carnosine concentrations and high intensity cycling capacity. Amino Acids. 2009;32(2):225-33. [PMID: 16868650]

39. Artioli GG, Gualano B, Smith A, Stout J, Lancha AH Jr. Role of beta-alanine supplementation on muscle carnosine and exercise performance. Med Sci Sports Exerc. 2010;42(6):1162-73. [PMID: 20479615]

40. Baguet A, Reyngoudt H, Pottier A, Everaert I, Callens S, et al Carnosine loading and washout in human skeletal muscles. J App Physiol. 2009;106(3):837-42. [PMID: 19131472]

41. Hoffman J, Ratamess N, Kang J, Mangine G, Faigenbaum A, et al Effect of creatine and beta-alanine supplementation on performance and endocrine responses in strength/power athletes. Int J Sport Nutr Exerc Metab. 2006;16(4):430-46. [PMID: 17136944]

42. Juneja LR, Chu DC, Okubo T, Nagato Y, Yokogoshi H. L-theanine-a unique acid of green tea and its relaxation effect in humans. Trends in Food Sci Tech. 2009;10(6):199-204.

43. Rogers $P$, Smith JE, Heatherley SV, Pleydell-Pearce CW. Time for tea: mood, blood pressure and cognitive performance effects of caffeine and theanine administered alone and together. Psychopharmacology. 2008;195(4):569-77. [PMID: 17891480]

44. Sinforiani E, Jannucceelli M, Mauri M, Costa A, Merlo P, et al Neuropsychological changes in demented patients treated with actyl-L-carnitine. Int J Clin Pharmacol Res. 1990;10(1-2):69-74. [PMID: 2387665]

45. Brooks JO, Yesavage JA, Carta A, Bravi D. Acetyl L-carnitine slows decline in younger patients with Alzheimer's disease: a reanalysis of a double-blind placebo-controlled study using the trilinear approach. Int Psychogeriatr. 1998;10(2):193-203. [PMID: 9677506]

46. Passeri M, lannuccelli M, Ciotti G, Bonati PA, Nolfe G, et al Mental impairment in aging: selection of patients, methods of evaluation and therapeutic possibilities of acetyl- L-carnitine. Int J Clin Pharmacol Res.1988;8(5):367-76. [PMID: 3229874]

47. Bass EP. Supplemental carnitine and exercise. Am J Clin Nutr. 2000;72(2 Suppl):618-23S. [PMID: 10919968]

48. Sun QQ, Xu SS, Pan JL, Guo HM, Cao WQ. Huperzine-A capsules enhance memory and learning in 34 pairs of matched adolescent students. Zhongguo Yao Li Xue Bao. 1999;20(7):601-3. [PMID: 10678121]

49. Kalman D, Feldman S, Samson A, Krieger D. A clinical evaluation to determine the safety, pharmacokinetics and pharmacodynamics of an inositol-stabilized arginine silicate dietary supplement in healthy adult males. Clin Pharmacol. 2015;7:103-9. [PMID: 26504409] 